\title{
Risk stratification system for biparametric prostate magnetic resonance imaging
}

\author{
Eugenio Martorana ${ }^{1}$, Pietro Scialpi ${ }^{2}$, Riccardo Grisanti $^{1}$, Michele Scialpi $^{3}$ \\ ${ }^{1}$ Department of Urology, Sassuolo Hospital, Sassuolo, Italy; ${ }^{2}$ Department of Urology, Portogruaro Hospital, Portogruaro, Italy; ${ }^{3}$ Division of \\ Diagnostic Imaging, Department of Surgical and Biomedical Sciences, University of Perugia, Italy \\ Correspondence to: Dr. Eugenio Martorana. Department of Urology, Sassuolo Hospital, Via Francesco Ruini, 2, Sassuolo, Italy. Email: \\ eugeniomartorana@gmail.com. \\ Provenance: This is an invited article commissioned by Section Editor Xiao Li (Department of Urology, Jiangsu Cancer Hospital \& Jiangsu Institute \\ of Cancer Research \& Nanjing Medical University Affiliated Cancer Hospital, Nanjing, China). \\ Comment on: Jambor I, Verho J, Ettala O, et al. Validation of IMPROD biparametric MRI in men with clinically suspected prostate cancer: A \\ prospective multi-institutional trial. PLoS Med 2019;16:e1002813.
}

Submitted Sep 09, 2019. Accepted for publication Sep 18, 2019.

doi: $10.21037 /$ tau. 2019.09 .34

View this article at: http://dx.doi.org/10.21037/tau.2019.09.34

We have read with great interest and appreciate the article by Jambor and colleagues published in the June 2019 issue of PLoS Med (1).

We congratulate the authors on their efforts to highlight the value of the biparametric magnetic resonance imaging (bpMRI) for the set of significant prostate cancer (PCa) diagnosis. Comparison between $\mathrm{bpMRI}$ and multiparametric MRI (mpMRI) of the prostate and results have already been reported in our previous experience (2) reporting no difference between the two protocols in terms of $\mathrm{PCa}$ detection and lesion localization within the prostate gland. Furthermore, we underline the uselessness of gadolinium and the risks of its accumulation in the basal nuclei whose potential side effects are still unknown (3). The use of gadolinium should only be used when strictly necessary.

Jambor et al.'s work, despite its validity, requires some clarifications. The authors adopt the Likert scale to attribute a risk score to all suspicious lesions included in the study. This risk score has been superseded by the most recent and widespread PIRADS v2 and PIRADS v2.1 score. So, we believe that Authors results are less comparable with that of other institutions. Moreover, Likert score, as well as PIRADS scores, include the use of gadolinium-based contrast agents. For this reason, it is difficult for us to understand how the Authors managed MRI findings to attribute a risk score by working with bpMRI (which does not involve the use of contrast enhancement). The lack of a specific bpMRI scoring system was the most important limits for bpMRI universal adoption. We focused our efforts on building a risk score adapted to the bpMRI exam: the simplified PIRADS score (S-PIRADS score) (4). The S-PIRADS is a risk score based on four categories according to lesions management:

* Category 1 and 2 whose management is similar to that proposed for PI-RADSv2 score 1-2 lesions;

* Category 3a includes focal rounded, lenticular, or irregular lesions, heterogeneous or homogeneous mild/moderately or markedly hypointense on T2-weighted (T2W), hyperintense on diffusionweighted images (DWI) with high b values and moderately hypointense on apparent diffusion coefficient (ADC) map with volume less than $0.5 \mathrm{cc}$. These lesions are candidates for clinical surveillance (prostate-specific antigen level and repeat biparametric MRI within 12 months);

* Category 3 b includes lesions with 3a similar bpMRI characteristics but with a volume greater than or equal to $0.5 \mathrm{cc}$. These lesions are candidates for targeted biopsy;

* Category 4 includes focal rounded, lenticular, or lesions, heterogeneous or homogeneous mild/ moderately or markedly hypointense on $\mathrm{T} 2 \mathrm{~W}$, hyperintense on DWI with high b values and markedly hypointense on ADC map in which the target biopsy check is indicated.

Finally, we disagree with the Authors about the transrectal biopsy approach. Firstly, because the rates of infection and 
the potential rectal bleeding make the use of this access unsafe. Secondly, because the inappropriate sampling of the apex and anterior zone lesions do not make the use of transrectal access universally adequate. In our experience we use the transperineal access adopting the Ginsburg Study Group scheme (5) and performing fusion biopsy on an axial plane. In our opinion, this approach makes the ultrasound localization of suspected area easier both on the axial and sagittal plane of the gland allowing the operator to perform targeted biopsy more accurately (especially in the cognitive modality). Using this approach we found a very low detection of clinically significant PCa $(\sim 2.8 \%)$ for S-PIRADS score 3 a lesions (volume $<0.5 \mathrm{cc}$ ) and a high detection of clinically significant PCa for S-PIRADS score $3 \mathrm{~b}$ (27.5\%) and S-PIRADS score 4 lesions $(77.1 \%)$ respectively.

In conclusion, we believe that bpMRI will become the main radiological tool in PCa diagnostic setting. Further efforts are still needed for the identification or validation of a universal bpMRI risk score.

\section{Acknowledgments}

None.

\section{Footnote}

Conflicts of Interest: The authors have no conflicts of interest to declare.

Cite this article as: Martorana E, Scialpi P, Grisanti R, Scialpi M. Risk stratification system for biparametric prostate magnetic resonance imaging. Transl Androl Urol 2019;8(Suppl 5):S482S483. doi: 10.21037/tau.2019.09.34
Ethical Statement: The authors are accountable for all aspects of the work in ensuring that questions related to the accuracy or integrity of any part of the work are appropriately investigated and resolved.

\section{References}

1. Jambor I, Verho J, Ettala O, et al. Validation of IMPROD biparametric MRI in men with clinically suspected prostate cancer: A prospective multi-institutional trial. PLoS Med 2019;16:e1002813.

2. Scialpi M, Prosperi E, D'Andrea A, et al. Biparametric versus Multiparametric MRI with Non-endorectal Coil at 3T in the Detection and Localization of Prostate Cancer. Anticancer Res 2017;37:1263-71.

3. Scialpi M, Rondoni V, Aisa MC, et al. Is contrast enhancement needed for diagnostic prostate MRI? Transl Androl Urol 2017;6:499-509.

4. Scialpi M, Aisa MC, D'Andrea A, et al. Simplified Prostate Imaging Reporting and Data System for Biparametric Prostate MRI: A Proposal. AJR Am J Roentgenol 2018;211:379-82.

5. Kuru TH, Wadhwa K, Chang RT, et al. Definitions of terms, processes and a minimum dataset for transperineal prostate biopsies: a standardization approach of the Ginsburg Study Group for Enhanced Prostate Diagnostics. BJU Int 2013;112:568-77. 